\title{
Collective Effects in High-Energy Muon Colliders
}

\author{
Eberhard Keil
}

\begin{abstract}
Collective single-beam effects, driven by impedances and wake fields in the vacuum chamber of high-energy muon colliders HEMC, are evaluated: (i) using techniques that have been applied to proton-proton colliders such as the LHC and extrapolations from it, (ii) using a new technique more applicable to nearly-isochronous HEMC, adding longitudinal and transverse kicks from longitudinal and transverse loss factors. Results from both techniques are presented for HEMC at 10 and $100 \mathrm{TeV}$ centre-of-mass energies.
\end{abstract}

Presented at Studies on Colliders and Collider Physics at the Highest Energies: Muon Colliders at $10 \mathrm{TeV}$ to $100 \mathrm{TeV}$, Montauk, New York, USA, 27 September to 1 October 1999

Geneva, Switzerland

February 8, 2000 


\section{INTRODUCTION}

In this contribution, I evaluate collective effects in high energy muon colliders HEMC in two different styles:

1. In the Section entitled "An-isochronous HEMC" I use a style similar to that used for large proton storage rings, e.g. LHC and VLHC. I present tables comparing collective effects in LHC and a high energy muon collider at $10 \mathrm{GeV}$ centre-ofmass energy, and a VLHC and a high energy muon collider at $100 \mathrm{GeV}$ centreof-mass energy, respectively.

2. In the Section entitled "Isochronous HEMC" I assume that the HEMC is isochronous, and that longitudinal motion is absent. I derive criteria for the longitudinal and transverse loss factors.

\section{AN-ISOCHRONOUS HEMC}

An-isochronous HEMC are similar to the LHC [1] and the VLHC [2, 3, 4]. The arcs consist of FODO cells, the momentum compaction does not vanish, and an RF system keeps the muon beams bunched. The resistive wall instability, coherent synchrotron tune shift, longitudinal microwave instability, and transverse mode coupling instability TMCI are important for LHC [5] and for the VLHC [6], and might be important for the HEMC. I first list the formulae that I shall use.

The growth rate of the resistive wall instability $\tau_{w}^{-1}$ is [5]:

$$
\tau_{w}^{-1}=\frac{r_{c} \bar{\beta} I F_{w}}{e Z_{0} \gamma b^{3}} \sqrt{\frac{\mu_{0} \rho_{w} C c}{\pi(n-Q)}}
$$

Here, $r_{c}$ is the classical muon radius, $\bar{\beta} \approx R / Q$ is the average $\beta$-function, $I$ is the total beam current, $Z_{0}$ is the impedance of free space, $\gamma$ is the usual relativistic factor, $b$ is the radius of the beam screen, $\rho_{w}$ is the resistivity of the beam screen, $C$ is the circumference, $c$ is the speed of light, and $(n-Q)=0.25$ is the tune of the $n$-th mode. The wall penetration factor $F_{w}$ describes the effects of a beam screen similar to that in the LHC [1], consisting of a thin inner $\mathrm{Cu}$ layer and a thicker layer of stainless steel [7].

To preserve longitudinal Landau damping, the synchrotron tune shift must remain smaller than the synchrotron tune spread. This leads to an upper limit for the imaginary part of the effective longitudinal impedance [6]:

$$
\Im\left(\frac{Z_{\mathrm{L}}}{n}\right)_{\text {eff }} \leq \frac{6}{\pi^{3}} \frac{h_{\mathrm{RF}}^{3} V_{\mathrm{RF}}}{I_{b}}\left(\frac{2 \pi \sigma_{s}}{C}\right)^{5}
$$

Here, $h_{\mathrm{RF}}$ and $V_{\mathrm{RF}}$ are the harmonic number and peak voltage of the RF system, $I_{b}$ is the bunch current, and $\sigma_{s}$ is the bunch length. All effective impedances $Z_{\text {eff }}$ are the weighted sums of $Z(\omega)$ times the bunch power spectrum [5]. 
The bunches are stable against the longitudinal microwave instability if the following inequality holds $[5,6]$ :

$$
\left|\left(\frac{Z_{\mathrm{L}}}{n}\right)_{\mathrm{eff}}\right| \leq \frac{12}{\pi^{3}} \frac{h_{\mathrm{RF}} V_{\mathrm{RF}}}{I_{b}}\left(\frac{2 \pi \sigma_{s}}{C}\right)^{3}
$$

The transverse mode coupling instability is caused by the shift of the $m=0$ headtail mode towards the $m=-1$ mode due to the broad band transverse impedance. The stability condition is $[5,6]$ :

$$
\Im\left(Z_{\mathrm{T}}\right)_{\mathrm{eff}} \leq \frac{8 Q_{s}}{\bar{\beta} I_{b}} \frac{2 \pi \sigma_{s}}{C} \frac{E}{e}
$$

Here, $Q_{s}$ is the synchrotron tune, and $E$ is the energy of the circulating muon beam.

\subsection{Comparison between LHC and HEMC10}

Tab. 1 shows the LHC and HEMC10 parameters needed for evaluating (1) to (4). I generated the LHC parameters at collision energy in a Mathematica notebook [8] that I have used for testing packages for the design of storage rings [9]. They are close to the official LHC parameters [1]. Similarly, I generate many of the HEMC10 parameters in a Mathematica notebook [10], and replace them by parameters in B. King's Parameter Table [11] before I evaluate (1) to (4).

Table 1: LHC and HEMC10 Parameters

\begin{tabular}{lrr}
\hline Parameter & LHC & HEMC10 \\
\hline CoM energy $E / \mathrm{TeV}$ & 14 & 10 \\
Circumference $C / \mathrm{m}$ & 26658 & 14916 \\
Average $\beta$-function $\bar{\beta} / \mathrm{m}$ & 68.1 & 31.8 \\
Momentum compaction $\eta$ & $3.1 \cdot 10^{-4}$ & $4 \cdot 10^{-5}$ \\
Vacuum chamber radius $b / \mathrm{mm}$ & 19 & 19 \\
Vacuum chamber material & $\mathrm{Cu} / \mathrm{Fe}$ & $\mathrm{W}$ \\
Vacuum chamber temperature/K & 20 & 300 \\
Vacuum chamber resistivity $\rho_{w} / \mathrm{n} \Omega \mathrm{m}$ & 0.55 & 55 \\
Harmonic number $h_{\mathrm{RF}}$ & 35560 & 24871 \\
Peak RF voltage $V_{\mathrm{RF}} / \mathrm{MV}$ & 16 & 100 \\
Synchrotron tune $Q_{s}$ & 0.00202 & 0.0018 \\
Bunch current $I_{b} / \mathrm{mA}$ & 0.188 & 9.8 \\
Beam current $I / \mathrm{mA}$ & 669 & 68.4 \\
Bunch length $\sigma_{s} / \mathrm{mm}$ & 75 & 2.2 \\
RMS relative energy spread $\sigma_{e} / 10^{-3}$ & 0.11 & 0.6 \\
\hline
\end{tabular}

Tab. 2 shows a comparison of the results for growth rates and thresholds for the LHC and HEMC10. In the LHC at injection energy, the resistive wall growth rate 
is larger, and the threshold impedance of the transverse mode-coupling instability is lower than the values at collision energy. The resistive-wall growth rate in HEMC10 is smaller than in the LHC. The three impedance thresholds are all worse to much worse in the HEMC10 than in the LHC. The reason for this is the fact that the bunch length in the HEMC10 has to be much smaller than in the LHC, in order to permit the very small value of the $\beta$-function at the interaction point. Note also that the number of synchrotron oscillations in a muon life time is of order unity.

Table 2: Comparison of growth rates and threshold impedances between LHC and HEMC10. The machine and beam parameters.are shown in Tab. 1

\begin{tabular}{lrr}
\hline & LHC & HEMC10 \\
\hline Resistive wall growth rate $\tau_{w}^{-1} / \mathrm{s}^{-1}$ & 6.6 & 2.6 \\
Coh. synchrotron tune shift $\Im\left(Z_{\mathrm{L}} / n\right)_{\text {eff }} / \Omega$ & 1.3 & $15 \cdot 10^{-6}$ \\
Long. $\mu$-wave instability $\left|\left(Z_{\mathrm{L}} / n\right)_{\text {eff }}\right| / \Omega$ & 6.45 & $4 \cdot 10^{-3}$ \\
TMCI threshold $\Im\left(Z_{\mathrm{T}}\right)_{\text {eff }} / \mathrm{M} \Omega \mathrm{m}^{-1}$ & 160 & 1.9 \\
\hline
\end{tabular}

In the LHC, the wall resistivity is approximately doubled by the $10 \%$ of the circumference with a $\mathrm{Cu}$ vacuum chamber at room temperature [5]. I ignore this factor in the calculation of the growth rates.

\subsection{Comparison between VLHC and HEMC100}

Tab. 3 shows the VLHC and HEMC100 parameters needed for evaluating (1) to (4). I generate also the VLHC and HEMC100 parameters in Mathematica notebooks [12, 13]. Again, I replace several parameters by parameters in B. King's Parameter Table [11] before I evaluate (1) to (4). In HEMC100, the synchrotron radiation damping time is of the order of the muon life time. Compensating the synchrotron radiation loss needs a substantial RF voltage. It remains to be checked whether avoiding the quantum excitation of the transverse emittance requires an arc lattice such that the equilibrium emittance is in the neighbourhood of the assumed one [4].

Tab. 4 shows a comparison of the results for growth rates and thresholds for the VLHC and HEMC100. The resistive-wall growth rate in HEMC100 is smaller than in the VLHC. The three impedance thresholds are all worse to much worse in the HEMC10 than in the LHC. The reason for this is again the fact that the bunch length in the HEMC100 has to be much smaller than in the VLHC. Contrary to HEMC10, the number of synchrotron oscillations in a muon life time is much larger than unity. 
Table 3: VLHC and HEMC100 Parameters

\begin{tabular}{lrr}
\hline Parameter & VLHC & HEMC100 \\
\hline CoM energy $E / \mathrm{TeV}$ & 100 & 100 \\
Circumference $C / \mathrm{km}$ & 120 & 105 \\
Average $\beta$-function $\bar{\beta} / \mathrm{m}$ & 542 & 255 \\
Momentum compaction $\eta$ & $8.9 \cdot 10^{-4}$ & $2.4 \cdot 10^{-4}$ \\
Vacuum chamber radius $b / \mathrm{mm}$ & 30 & 19 \\
Vacuum chamber material & $\mathrm{Cu} / \mathrm{Fe}$ & $\mathrm{W}$ \\
Vacuum chamber temperature $/ \mathrm{K}$ & 20 & 300 \\
Vacuum chamber resistivity $\rho_{w} / \mathrm{n} \Omega \mathrm{m}$ & 0.55 & 55 \\
Harmonic number $h_{\mathrm{RF}}$ & 159960 & 104825 \\
Peak RF voltage $V_{\mathrm{RF}} / \mathrm{MV}$ & 7 & 25000 \\
Synchrotron tune $Q_{s}$ & 0.00165 & 0.026 \\
Bunch current $I_{b} / \mu \mathrm{A}$ & 7.2 & 277 \\
Beam current $I / \mathrm{mA}$ & 231 & 6.9 \\
Bunch length $\sigma_{s} / \mathrm{mm}$ & 47.3 & 2.5 \\
RMS relative energy spread $\sigma_{e} / 10^{-3}$ & 0.0046 & 0.113 \\
\hline
\end{tabular}

Table 4: Comparison of growth rates and threshold impedances between VLHC and HEMC100. The machine and beam parameters.are shown in Tab. 3

\begin{tabular}{lrr}
\hline & VLHC & HEMC100 \\
\hline Resistive wall growth rate $\tau_{w}^{-1} / \mathrm{s}^{-1}$ & 3 & 0.14 \\
Coh. synchrotron tune shift $\Im\left(Z_{\mathrm{L}} / n\right)_{\text {eff }} / \mathrm{m} \Omega$ & 28 & 0.003 \\
Long. $\mu$-wave instability $\mid\left(Z_{\mathrm{L}} / n\right)_{\text {eff }} / \Omega$ & 0.63 & 0.017 \\
TMCI threshold $\Im\left(Z_{\mathrm{T}}\right)_{\text {eff }} / \mathrm{M} \Omega \mathrm{m}^{-1}$ & 420 & 28 \\
\hline
\end{tabular}

\section{ISOCHRONOUS HEMC}

In an isochronous HEMC, synchrotron oscillations are absent, and the positions of the muons along the bunch are frozen, whatever RF accelerating fields and longitudinal and transverse wake fields they see. The longitudinal wake field changes the energy of the muons.

In the longitudinal direction, I derive the following inequality for the longitudinal loss factor $k_{\|}$by imposing the condition that the energy change accumulated over the relativistic muon lifetime $T_{\mu}$, expressed as the number of turns $n_{\text {turn }}$, is not larger than the rms beam energy spread $E \sigma_{e} / 2$ :

$$
k_{\|} \leq \frac{(E / e) \sigma_{e}}{2 N e n_{\text {turn }}}=\frac{(E / e) \sigma_{e}}{2 I_{b} T_{\mu}}
$$


Here, $E$ is the centre-of-mass energy of the muons, i.e. twice the muon beam energy, $e$ is the muon charge, $\sigma_{e}$ is the relative rms energy in the beam, $N$ is the bunch population, and $I_{b}$ the bunch current. Strictly speaking, $k_{\|}$should be interpreted as the variation of the loss factor along the bunch. That part of $k_{\|}$which is constant along the bunch simply changes the energy of all muons, but does not contribute to the energy spread.

In the transverse direction, I derive the following inequality for the transverse loss factor $k_{\perp}$ by imposing the condition that the transverse kicks accumulated over the relativistic muon lifetime, expressed as the number of turns $n_{\text {turn }}$, are not larger than the rms beam divergence at the interaction point $\sigma^{\prime}$ :

$$
k_{\perp} \leq \frac{(E / e)}{2 N e n_{\text {turn }} \bar{\beta}}=\frac{(E / e)}{2 I_{b} T_{\mu} \bar{\beta}}
$$

Here, $\bar{\beta}$ is the average value of the $\beta$-function in the components that drive $k_{\perp}$. There are no coherent transverse kicks when the beam is perfectly centred in the vacuum chamber. I therefore assumed that the typical offset of the beam around the circumference is of the order of the rms beam radius there in order to derive (6). The transverse kicks have two effects on the beams. They displace the beam centres, thus eventually causing mis-crossings at the interaction points, while their variation along the bunch causes an growth of the emittance.

Tab. 5 shows the maximum tolerable loss factors $k_{\|}$and $k_{\perp}$, found by applying (5) and (6) to HEMC10 and HEMC100, respectively. I leave to the reader a comparison between these values, and the values of the loss factors that are likely to occur in HEMC10 and HEMC100.

Table 5: Maximum tolerable loss factors $k_{\|}$and $k_{\perp}$ in HEMC10 and HEMC100

\begin{tabular}{lrr}
\hline & HEMC10 & HEMC100 \\
\hline CoM energy E/TeV & 100 & 100 \\
RMS relative energy spread $\sigma_{e} / 10^{-3}$ & 0.0046 & 0.113 \\
Average $\beta$-function $\bar{\beta} / \mathrm{m}$ & 542 & 255 \\
Muon life time $n_{\text {turn }}$ & 2090 & 2975 \\
\hline Longitudinal loss factor $k_{\|} / \mathrm{VpCb}^{-1}$ & 2.94 & 19.7 \\
Transverse loss factor $k_{\perp} / \mathrm{V}(\mathrm{pCbm})^{-1}$ & $4.91 \cdot 10^{3}$ & $1.74 \cdot 10^{5}$ \\
\hline
\end{tabular}

\section{References}

[1] The LHC Study Group, CERN/AC/95-05 (LHC) (1995).

[2] G. Dugan et al., Proc. 1996 DPF/DPB Summer Study on New Directions in HighEnergy Physics (1997) 251, also Cornell University CBN 96-18 (1996). 
[3] E. Keil, CERN/SL/97-13 (AP) (1997).

[4] E. Keil, Proc. Particle Accelerator Conference PAC'97 (Vancouver, 12-16 May 1997) 104; also CERN-LHC-PROJECT-REPORT-102.

[5] F. Ruggiero, Particle Accelerators 50 (1995) 83.

[6] J.T. Rogers, Proc. 1996 DPF/DPB Summer Study on New Directions in HighEnergy Physics (1997) 337, also Cornell University CBN 96-14 (1996).

[7] E. Keil and B. Zotter, Proc. 6th European Particle Accelerator Conference EPAC'98 (Stockholm, 22-26 June 1998) 963; also CERN-SL-98-021-AP.

[8] E. Keil, http://wwwslap.cern.ch/ keil/Math/lhc.nb

[9] E. Keil, CERN-SL-99-053-AP (1999).

[10] E. Keil, http://wwwslap.cern.ch/ keil/Math/hemc10TeV.nb

[11] B. King, http://pubweb.bnl.gov/people/bking/heshop/hemc_para.html

[12] E. Keil, http://wwwslap.cern.ch/ keil/Math/vlhc50GeV.nb

[13] E. Keil, http://wwwslap.cern.ch/ keil/Math/hemc100TeV.nb 